Supporting Information

\title{
Fabrication of Field-Effect Transistors from Hexathiapentacene Single-Crystal Nanowires
}

\author{
Alejandro L. Briseno, ${ }^{1, *}$ Stefan C. B. Mannsfeld, ${ }^{2}$ Xianmao Lu, ${ }^{1}$ Yujie Xiong, ${ }^{1}$ Samson A. Jenekhe,,${ }^{1,3}$ \\ Zhenan Bao, ${ }^{2^{*}}$ and Younan $\mathrm{Xia}^{1, *}$ \\ ${ }^{1}$ Department of Chemistry, University of Washington, Seattle, Washington 98195 \\ ${ }^{2}$ Department of Chemical Engineering, Stanford University, Stanford, California 94305 \\ ${ }^{3}$ Department of Chemical Engineering, University of Washington, Seattle, Washington 98195 \\ E-mail:xia@chem.washington.edu; abriseno@u.washington.edu; zbao@stanford.edu
}

1. Synthesis of HTP nanowires. The synthesis and X-ray crystal structure of hexathiapentacene are reported elsewhere. ${ }^{1}$ Briefly, pentacene $(400 \mathrm{mg})$ and sulfur $(400$ $\mathrm{mg}$ ) were degassed and heated to reflux in $30 \mathrm{~mL}$ of 1,2,4-trichlorobenzene under nitrogen. After refluxing for 3 hours a hot filtration was immediately performed to pass impurities and side products in the hot 1,2,4-trichlorobenzene. The crystalline product was washed with acetone and ethanol. The product was dried in an oven at $70{ }^{\circ} \mathrm{C}$ and weighed ( $\sim 35-40 \%$ product yield). HTP nanowire synthesis: to a $50 \mathrm{~mL}$ round-bottom flask add $15 \mathrm{~mL}$ of benzonitrile or other high boiling solvents (e.g., nitrobenzene or ODCB) and add $\sim 0.018 \mathrm{~g}$ of HTP powder. Stir the solution with a magnetic device and bring the mixture to boiling until all the powder completely dissolves and continue to heat for an additional 5 minutes. One can control the dimensions of the nanowires by adding methanol from a dropping funnel as shown in the ensuing schematic (S1). Do not add the methanol until the heat is completely turned off and the heating mantle device is removed from underneath the flask. Caution: be certain to perform the experiment in a well-ventilated fume hood and utilize a Plexiglas guard shield for protection as addition of the methanol to the hot benzonitrile can boil over and vigorously splash. It is suggested to utilize an over-sized flask to minimize the boil-over effect. Single-crystal nanowires immediately begin to form and within an hour a cottonlike state can be collected from the organic solvent and transferred to chloroform or any other organic solvent of interest.

\section{Reference}

1. Briseno, A. L.; Miao, Q.; Ling, M.-M.; Reese, C.; Meng, H.; Bao, Z.; Wudl, F. J. Am. Chem. Soc., 2006, 128, 15576-15577. 


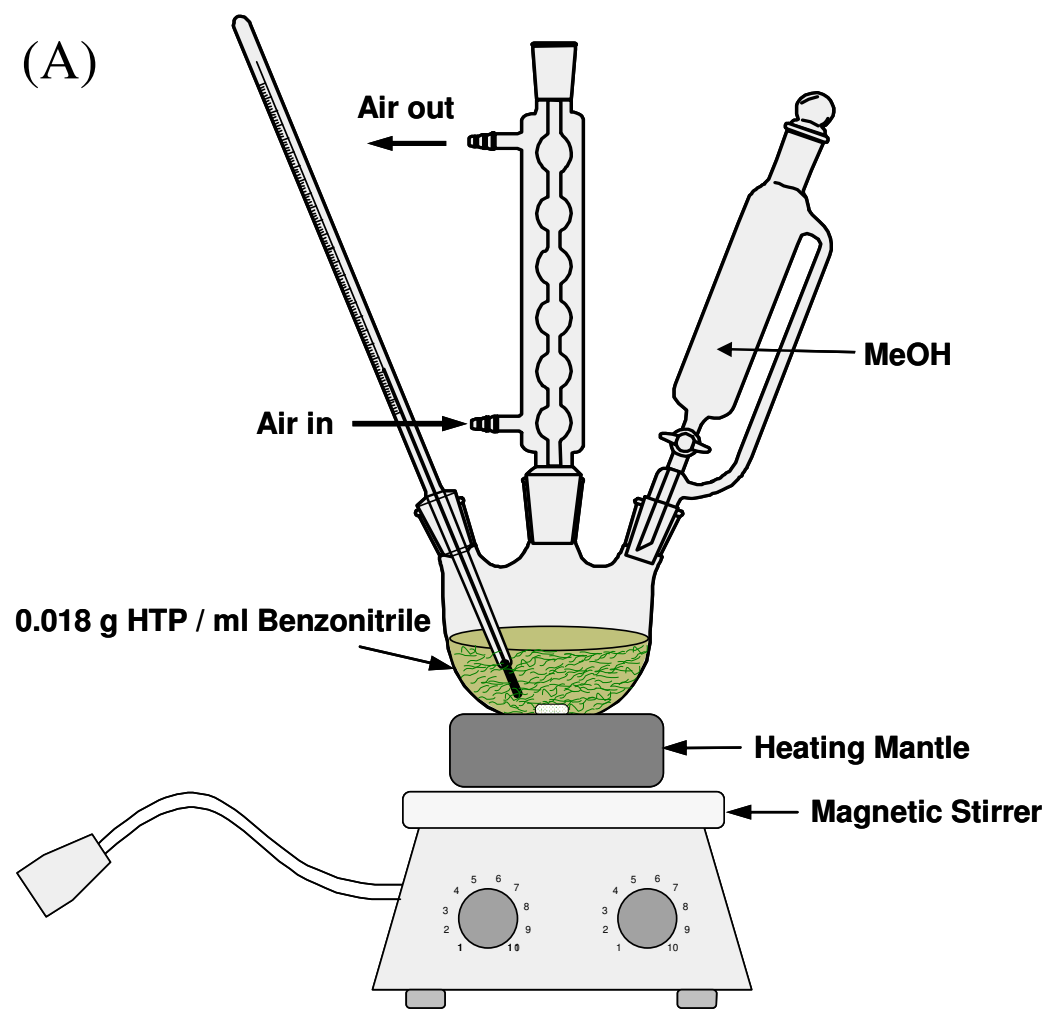

(B)

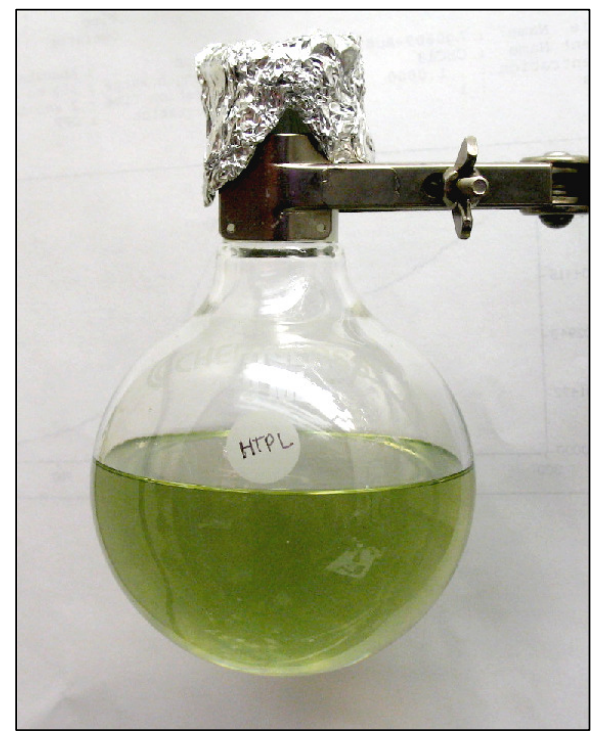

Figure S1. (A) Experimental setup for the synthesis of HTP nanowires, and (B) a digital photo of a bulk HTP synthesis. The solution-phase route enables the synthesis of bulk quantities of HTP nanowires in one step. 
2. Device Fabrication. Single-nanowire transistors were fabricated on $300 \mathrm{~nm} \mathrm{SiO}_{2}$ dielectric substrates on heavily doped silicon. Bottom contact source-drain electrodes $(\mathrm{Cr}=2 \mathrm{~nm}, \mathrm{Au}=40 \mathrm{~nm})$ were fabricated by conventional photolithography using a transparency photomask. Due to the processability of the HTP nanowires in various solvents, we prepared functional transistors via drop-casting, spin-coating, and spraycoating from very dilute HTP dispersions in chloroform or ethanol. Flexible transistor devices were fabricated onto $140 \mu \mathrm{m}$ Kapton (polyimide) substrates coated with $100 \mathrm{~nm}$ $\mathrm{Au}$. Polymer dielectric layer was spin-coated at $\sim 2,000 \mathrm{rpm}$ from a mixture of $22 \mathrm{wt} \%$ polyvinylphenol (PVP, MW=20,000) and $+8 \mathrm{wt} \%$ poly(melamine-co-formaldehyde) methylated (MW=511) crosslinker in propylene glycol methyl ether acetate (PGMEA) using the procedure reported in a previous paper (Briseno et al., Adv. Mater. 2006, 18, 2320-2324). Typical dielectric thickness was $1.5 \mu \mathrm{m}$ and a capacitance of $1.9 \mathrm{nF} / \mathrm{cm}^{2}$ was measured. Shadow masks were used to evaporate $\mathrm{Cr} / \mathrm{Au}$ for source-drain contacts. Electrical measurements were conducted using a Keithley 4200 SC semiconductor analyzer. In situations where more than one wire bridged the source-drain electrodes, a micromanipulator from the probe station was used to break away the wire. After measuring devices, we typically conducted SEM or optical microscope measurements to acquire accurate nanowire dimensions. The $W / L$ for nanowire transistors ranged from approximately 0.04 to 0.065 with an average value of 0.059 . We note that mobility values were calculated on individual $W / L$ values. For network transistors, we multiplied the number of nanowires bridging source-drain electrodes times 0.059 . However, for devices containing a large density of nanowires, we used the width of the contact pads as $W$ since trying to count the number of nanowires became difficult. We note that nanowire devices failed to function after SEM imaging (likely due to the electron beam-induced damage to the semiconductor). Strain $(S)$ calculations were determined from the given equation,

$$
S=\left(\frac{d f+d s}{2 R}\right) \frac{\left(1+2 \eta+\chi \eta^{2}\right)}{(1+\eta)(1+\chi \eta)}
$$

where $R$ is the bending radius, $d_{\mathrm{f}}$ the thickness of a gate dielectric, $d_{\mathrm{s}}$ the substrate thickness, $\eta=d_{\mathrm{f}} / d_{\mathrm{s}}, \chi=Y_{\mathrm{f}} / Y_{\mathrm{s}}: Y_{\mathrm{f}}$ and $Y_{\mathrm{s}}$ Young's modulus of the gate dielectric and the substrate, respectively. 
3. Thickness of the conducting channel and validity of sheet capacitance approximation. An important question is whether or not the relatively small diameter (W) of organic nanowires can satisfy the sheet capacitance approximation and be a reliable estimator of mobility. It is known from theoretical calculations and experiments on similar organic semiconductor materials [1-3] that the charge density populating the semiconductor layer in the transistor's on-state (large gate voltage) is confined to the first few monolayers above the insulator/semiconductor interface (for alpha-6T it has dropped a factor of 10 already $2 \mathrm{~nm}$ away from the interface to the dielectric). In addition, it is presumed that the charges located near the interface have the highest mobility. The charge-sheet approximation requires that the charge density distribution in the conducting channel can be approximated by a sheet of charges whose height is much smaller than its lateral dimension (which is $\mathrm{W}$ ) and the gate oxide thickness. HTP nanowires range from $200 \mathrm{~nm}$ to about $1 \mu \mathrm{m}$ wide and assuming that there is a similar decline of charge density in the nanowire vertical to the channel $(\sim 2 \mathrm{~nm})$ under large gate biases, the height of the corresponding charge sheet is still much smaller than the gate dielectric layer thickness and the nanowire diameter (a factor of about 100 to 500). Therefore we conclude that in case of our organic nanowires the charge-sheet approximation still represents a relatively good estimate for the charge density distribution at the dielectric interface, just as it applies to a thin-film transistor.

\section{References}

[1] Alam et al., IEEE Trans. Elec. Dev. 1997, 144, 1332-1337.

[2] Tanase et al., Phys. Stat. Sol. 2004, 201, 1236-1245.

[3] Horowitz, G. J. Mater. Res., 2004, 19, 1946-1962. 


\section{Mechanical strain data of single-nanowire FETs.}
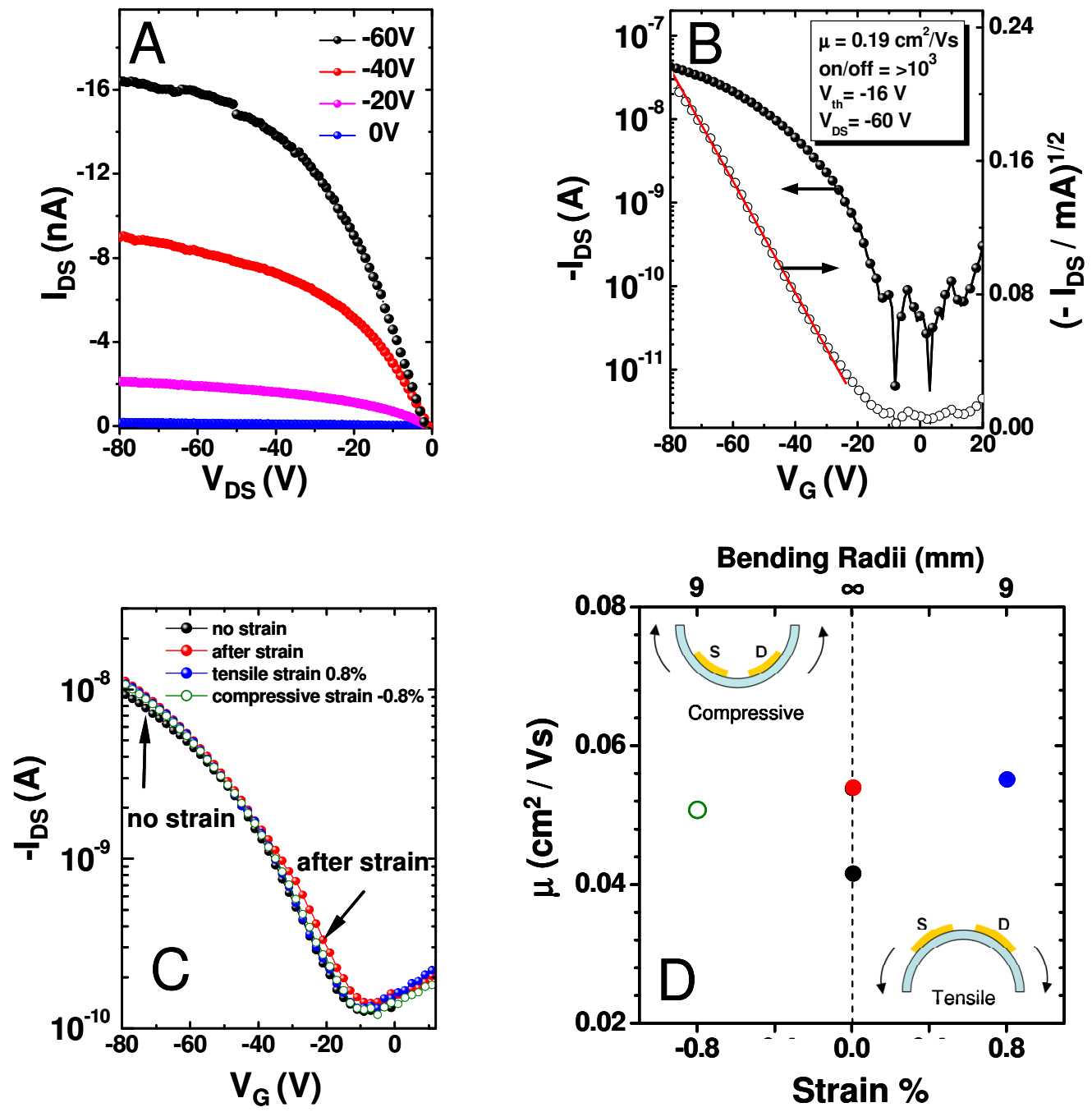

Figure S2. (A) Output characteristics and (B) transfer characteristics of an HTP singlenanowire transistor fabricated on a flexible Kapton substrate. (C) An overlay of sourcedrain currents $\left(\mathrm{I}_{\mathrm{DS}}\right)$ at different geometrical strain conditions. Note: experiments in figures $\mathrm{A}$ and $\mathrm{B}$ do not correspond to the device in figures $\mathrm{C}$ and $\mathrm{D}$. All scans conducted at $\mathrm{V}_{\mathrm{DS}}=-60 \mathrm{~V}$. (D) A plot of mobility versus bending radii (strain \%) for a single-wire transistor. The negative and positive strain \% corresponds to "compressive" and "tensile" bending, respectively. The lowest-most data point $\left(\sim 0.042 \mathrm{~cm}^{2} / \mathrm{Vs}\right)$ at zero strain $\%$ corresponds to the device mobility before any bending experiments were conducted. All points are color-coordinated to match the legends in figure $\mathrm{C}$. 


\section{Additional images of HTP nanowire devices.}
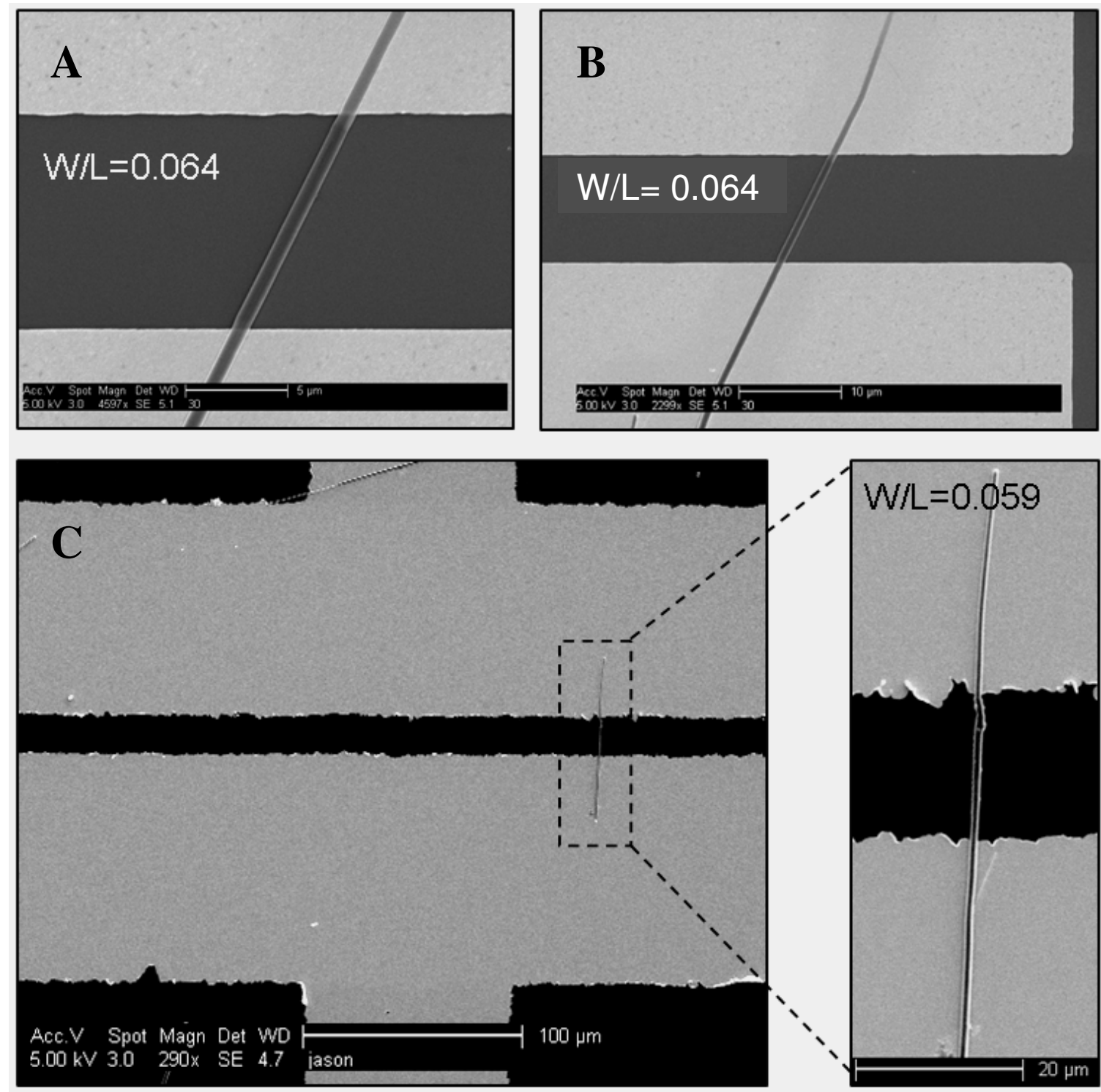

Figure S3. (A-C) Representative single-nanowire HTP field-effect transistors. HTP nanowire devices exhibited mobilities on the order of $10^{-1}$ to $10^{-2} \mathrm{~cm}^{2} / \mathrm{Vs}$. All devices were in the bottom-contact configuration. Panel $\mathrm{C}$ illustrates the actual device that generated the highest mobility of $0.27 \mathrm{~cm}^{2} / \mathrm{Vs}$ (see Figure $3, \mathrm{~A}$ and $\mathrm{B}$, for device characteristics). The slope for this device was $8.9 \times 10^{-6}, W / L=0.059, \mathrm{C} i=10 \mathrm{nF} / \mathrm{cm}^{2}$. 


\section{Large-area device fabrication.}

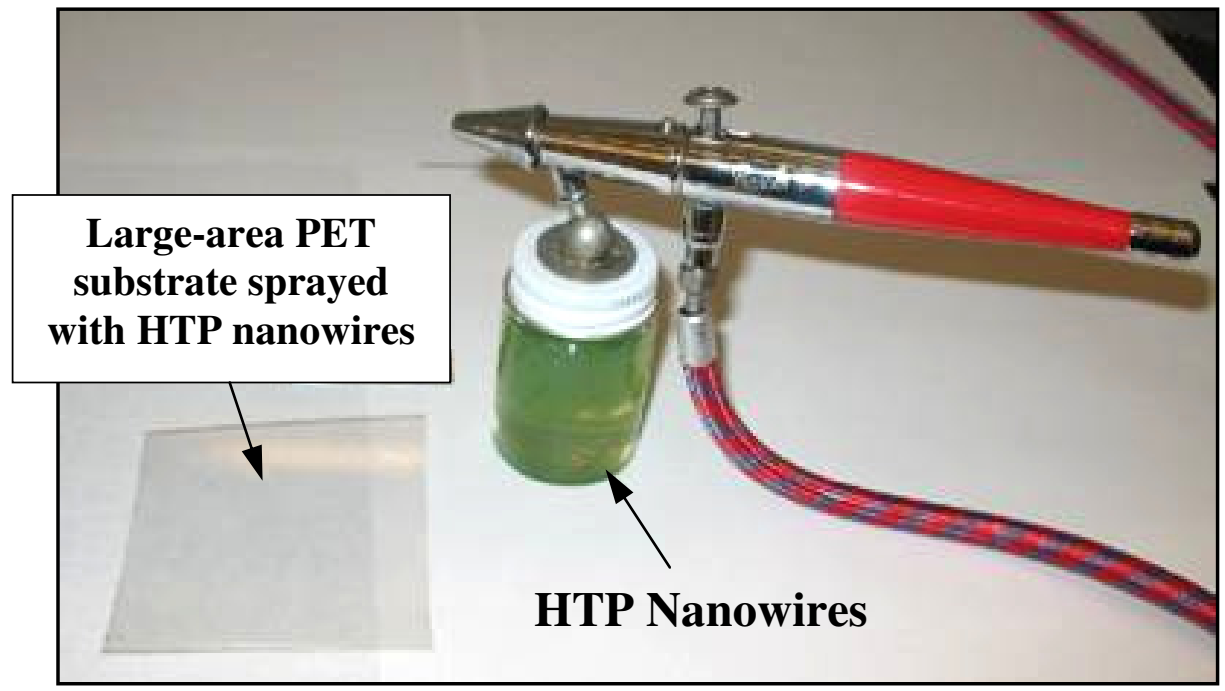

Figure S4. The airbrush device used to spray-coat HTP nanowires onto plastic substrates. The nanowires were dispersed in chloroform, ethanol or a mixture of these two solvents. 